\title{
Maximum Likelihood DOA Estimation of Multiple Wideband Sources in the Presence of Nonuniform Sensor Noise
}

\author{
C. E. Chen, F. Lorenzelli, R. E. Hudson, and K. Yao \\ Los Angeles EE Department, University of California, Los Angeles, CA 90095, USA \\ Correspondence should be addressed to C. E. Chen, chiaoen@ucla.edu
}

Received 1 March 2007; Revised 21 July 2007; Accepted 8 October 2007

Recommended by Sinan Gezici

We investigate the maximum likelihood (ML) direction-of-arrival (DOA) estimation of multiple wideband sources in the presence of unknown nonuniform sensor noise. New closed-form expression for the direction estimation Cramér-Rao-Bound (CRB) has been derived. The performance of the conventional wideband uniform ML estimator under nonuniform noise has been studied. In order to mitigate the performance degradation caused by the nonuniformity of the noise, a new deterministic wideband nonuniform ML DOA estimator is derived and two associated processing algorithms are proposed. The first algorithm is based on an iterative procedure which stepwise concentrates the log-likelihood function with respect to the DOAs and the noise nuisance parameters, while the second is a noniterative algorithm that maximizes the derived approximately concentrated loglikelihood function. The performance of the proposed algorithms is tested through extensive computer simulations. Simulation results show the stepwise-concentrated ML algorithm (SC-ML) requires only a few iterations to converge and both the SC-ML and the approximately-concentrated ML algorithm (AC-ML) attain a solution close to the derived CRB at high signal-to-noise ratio.

Copyright $\odot 2008$ C. E. Chen et al. This is an open access article distributed under the Creative Commons Attribution License, which permits unrestricted use, distribution, and reproduction in any medium, provided the original work is properly cited.

\section{INTRODUCTION}

Direction-of-arrival (DOA) estimation has been one of the central problems in radar, sonar, navigation, geophysics, and acoustic tracking. A wide variety of high-resolution narrowband DOA estimators have been proposed and analyzed in the past few decades [1-4]. The maximum likelihood (ML) estimator, which shows excellent asymptotic performance, plays an important role among these techniques. Many of the proposed ML estimators are derived from the uniform white noise assumption [4-6], in which the noise process of each sensor is assumed to be spatially uncorrelated white Gaussian with identical unknown variance. It is shown that under this assumption the ML estimates of the nuisance parameters (source waveforms/spectra and noise variance) can be expressed as a function of DOAs [7-9], and therefore the number of independent parameters to be estimated is reduced. This procedure is called concentration, which substantially reduces the search space and usually leads to a more efficient implementation.

Recently, there has been a great interest in estimating the DOAs for wideband sources, whose energy is spread over a broad bandwidth. For example, acoustic signals can range from $20 \mathrm{~Hz}$ to $20 \mathrm{kHz}$ depending on the type of sources. For wideband signals, many of the narrowband DOA estimation algorithms cannot be directly applied since the phase difference between sensor pairs depends on not only the DOAs but also the temporal frequencies. An intuitive way of generalizing a narrowband algorithm to wideband algorithm is to use the discrete Fourier transform (DFT) to decompose the signal into narrowband signals of different frequencies, apply the narrowband algorithms to each component, and fuse the overall estimation results. Better estimation accuracy is usually obtained when applying wideband algorithms to wideband sources since the processing gain from the frequency diversity is exploited. Various wideband ML estimators have been proposed in the literature [10-12], most of them are either derived under the uniform white noise assumption $[10,12]$ or assume the noise spectrum is known or estimated a priori [11].

The uniform white noise assumption is unrealistic in many applications. For densely placed sensors, the noise can be correlated and therefore should be modeled as a colored random process. In order to reduce the number of nuisance 
noise parameters, various noise modeling techniques have been proposed in the literature [13-15]. In [13] the noise is assumed to be an autoregressive moving-average (ARMA) process whose parameters need to be estimated from a preliminary step, while in [14] the noise covariance matrix is modeled as a sum of Hermitian matrices known up to a multiplicative scalar. In [16], a "despoking" technique is proposed based on the assumption that the noise field is invariant under two measurements of the array covariance. Closely related to the ML estimator, a wideband DOA estimator in the presence of colored noise has been proposed using the generalized-least-squares approach [17]. The main restriction of this method is that the signal spectral density matrices are assumed to be the same for all frequency bins, which does not hold for many wideband signals.

In several practical applications, the sensors are sparsely placed so that the sensor noise is spatially uncorrelated. However, the noise power of each sensor can still be different due to the variation of the manufacturing process or the imperfection of array calibration. As a result, the noise covariance matrix can be modeled as a diagonal matrix where the diagonal elements in general are not identical. It is crucial to note that this modeling is not a special case of the ARMA modeling [13], as is explained in [18]. Furthermore, the DOA estimators derived from either the uniform white noise assumption or the general colored noise modeling techniques may not give satisfactory results in this nonuniform uncorrelated noise environment since the algorithm derived from the uniform white noise assumption blindly treats all sensors equally and the general colored noise modeling technique neglects the fact that the sensor noise is uncorrelated. Motivated by the arguments above, a narrowband ML DOA estimator under this nonuniform sensor noise model has been recently derived [18]. Yet to the best of our knowledge, neither the nonuniform wideband ML DOA estimator under the same noise model nor its theoretical bound has ever been investigated in the literature.

In this paper, we derive a new wideband ML DOA estimator and a new closed-form expression of the CramérRao bound $(\mathrm{CRB})$ in the presence of unknown nonuniform noise. Our expression can be viewed as an extension of [12] to nonuniform noise scenarios and a generalization of [18] to a wideband signal model. It turns out that the derived nonuniform wideband ML DOA estimator cannot be concentrated analytically, and therefore the direct implementation would require an exhaustive search in the jointparameter space. In order to reduce the complexity, two different algorithms have been proposed. The first algorithm is based on an iterative procedure which stepwise concentrates the log-likelihood function, while the second is a noniterative algorithm that maximizes the derived approximatelyconcentrated (AC) log-likelihood function.

The rest of the paper is organized as follows. The wideband signal model is introduced in Section 2, and the conventional wideband uniform ML DOA estimator (uniformML estimator) [12] is reviewed in Section 3. In Section 4, the new wideband nonuniform ML estimator (nonuniform-ML estimator) is first derived, and two new algorithms are proposed. The derived CRB is also presented in the same section.
The performance of the proposed algorithms is studied and compared with the CRB through computer simulations and is shown in Section 5. The paper is concluded in Section 6.

Throughout this paper, the superscripts $T, *, H$, and $\dagger$ stand for the transpose, conjugate, conjugate transpose, and pseudoinverse of a matrix while $\otimes$ and $\odot$ stand for the Kronecker and Hadamard matrix product operators. The real part and imaginary part of a matrix are denoted by $\mathfrak{R}\{\cdot\}$ and $\mathfrak{I}\{\cdot\}$, respectively, while the Euclidean norm is denoted as $\|\cdot\|, \mathbf{1}$ is the vector of all ones and $\mathbf{I}$ is the identity matrix.

\section{WIDEBAND SIGNAL MODEL}

Let there be $M$ wideband sources in the far-field of a $P$ element arbitrarily distributed array $(M<P)$. For simplicity, we assume the sources and the array lie in the same plane, and $\theta_{m}$ denotes the DOA of the $m$ th source with respect to the centroid of the array, $m=1, \ldots, M$. The number of sources is assumed to be known or has been correctly estimated by [19]. Without loss of generality, we set the array centroid to be at the origin, and the position vector of each sensor is expressed as $\mathbf{r}_{p}=\left[r_{p} \cos \left(\phi_{p}\right), r_{p} \sin \left(\phi_{p}\right)\right]^{T}$, $p=1, \ldots, P$. For a uniform circular array (UCA), $\mathbf{r}_{p}$ can be further simplified to $\mathbf{r}_{p}=[R \cos (2 \pi(p-1) / P), R \sin (2 \pi(p-$ $1) / P)]^{T}$, where $R$ is the radius of the UCA. In this paper, we derive our wideband algorithms and the CRB for arrays of arbitrary geometry, while the simulation is performed under a UCA setting (Section 5).

For a general array geometry, the time-delay (in samples) from the $m$ th source to the $p$ th sensor relative to the centroid can be expressed as $t_{p}^{(m)}=r_{p} F_{s} \cos \left(\theta_{m}-\phi_{p}\right) / v$, where $F_{s}$ is the sampling frequency and $v$ is the known propagation speed of wave. The received waveform by the $p$ th sensor at time instant $n$ can then be expressed as

$$
x_{p}(n)=\sum_{m=1}^{M} s^{(m)}\left(n-t_{p}^{(m)}\right)+w_{p}(n),
$$

for $n=0, \ldots, N-1$. Here $N$ denotes the number of samples per frame, $s^{(m)}(n)$ is the signal waveform of the $m$ th source, and $w_{p}(n)$ is the sensor noise at the $p$ th sensor. We transform (1) into the frequency domain via the DFT, which allows the signal model to be expressed with multiplicative steering vectors instead of time delays.

After applying the $N$-point DFT to (1), we obtain the following signal model:

$$
\mathbf{X}(k)=\mathbf{D}(k) \mathbf{S}(k)+\mathbf{W}(k),
$$

where $k=0, \ldots, N-1$. Here $\mathbf{X}(k)=\left[X_{1}(k), \ldots, X_{P}(k)\right]^{T}$ denotes the spectrum of the observed waveform, and $\mathbf{S}(k)=$ $\left[S^{(1)}(k), \ldots, S^{(m)}(k)\right]^{T}$ and $\mathbf{W}(k)=\left[W_{1}(k), \ldots, W_{P}(k)\right]^{T}$ are the complex source and noise spectrum, respectively. $\mathbf{D}(k)=\left[\mathbf{d}^{(1)}(k), \ldots, \mathbf{d}^{(M)}(k)\right]$ is called the steering matrix, where $\mathbf{d}^{(m)}(k)=\left[d_{1}^{(m)}(k), \ldots, d_{p}^{(m)}(k)\right]^{T}$ is the steering vector of the $m$ th source. Let the sensor response from the $m$ th source to the $p$ th sensor be $a_{p}\left(k, \theta_{m}\right)$, then $d_{p}^{(m)}(k)=$ $a_{p}\left(k, \theta_{m}\right) e^{-j 2 \pi k t_{p}^{(m)} / N}$. 
Throughout this paper, we assume the source spectrum $\mathbf{S}(k)$ to be deterministic and unknown while the noise spectrum $\mathbf{W}(k)$ is modeled as a spatially uncorrelated zero-mean white Gaussian process with the following covariance matrix:

$$
\begin{aligned}
\mathbf{Q} & =E\left\{\mathbf{W}(k) \mathbf{W}(k)^{H}\right\} \\
& =\left[\begin{array}{llll}
q_{1} & & & 0 \\
& q_{2} & & \\
& & \ddots & \\
0 & & & q_{p}
\end{array}\right] .
\end{aligned}
$$

\section{REVIEW ON THE WIDEBAND MAXIMUM-LIKELIHOOD DOA ESTIMATOR IN THE PRESENCE OF UNIFORM SENSOR NOISE}

In this section, we review on the conventional uniform noise wideband ML DOA estimator (uniform-ML estimator) [12]. The estimator is derived from the wideband deterministic signal model (2) along with the uniform white noise assumption of $\mathbf{Q}=\sigma^{2} \mathbf{I}$.

Denote $\boldsymbol{\Omega}=\left[\boldsymbol{\Theta}^{T}, \mathbf{S}^{T}, \sigma^{2}\right]^{T}$ as the vector of all the unknown parameters in the model, where

$$
\begin{aligned}
& \boldsymbol{\Theta}=\left[\theta_{1}, \ldots, \theta_{M}\right]^{T}, \\
& \mathbf{S}=\left[\mathbf{S}(1)^{T}, \ldots, \mathbf{S}\left(\frac{N}{2}\right) T\right]^{T},
\end{aligned}
$$

then the likelihood function of $\boldsymbol{\Omega}$ can be expressed as

$$
f(\boldsymbol{\Omega})=\frac{1}{\pi^{P N / 2} \sigma^{P N}} \exp \left\{-\frac{1}{\sigma^{2}} \sum_{k=1}^{N / 2}\|\mathbf{g}(k)\|^{2}\right\},
$$

where

$$
\mathbf{g}(k)=\mathbf{X}(k)-\mathbf{D}(k) \mathbf{S}(k) .
$$

Take the logarithm of (5) and neglect all the constant terms, the log-likelihood function $L(\boldsymbol{\Omega})$ can be expressed as

$$
L(\boldsymbol{\Omega})=-\frac{P N}{2} \log \sigma^{2}-\frac{1}{\sigma^{2}} \sum_{k=1}^{N / 2}\|\mathbf{g}(k)\|^{2} .
$$

It follows that the maximum likelihood estimator for $\boldsymbol{\Omega}$ is simply

$$
\widehat{\mathbf{\Omega}}=\arg \max _{\boldsymbol{\Omega}} L(\boldsymbol{\Omega}) .
$$

It is clear that the dependency of $L(\boldsymbol{\Omega})$ with respect to $\boldsymbol{\Theta}$ and $\mathbf{S}$ is through $\|\mathbf{g}(k)\|^{2}$, which is independent of $\sigma^{2}$. It follows that a concentrated ML estimator for $\Theta$ and $\mathbf{S}$ can be obtained immediately by

$$
(\widehat{\boldsymbol{\Theta}}, \widehat{\mathbf{S}})=\arg \min _{\boldsymbol{\Theta}, \mathbf{S}} \sum_{k=1}^{N / 2}\|\mathbf{g}(k)\|^{2} .
$$

Here $\mathbf{S}(k)$ and $\boldsymbol{\Theta}$ are referred to as the linear and nonlinear parameters, respectively, since the noise corrupted data $\mathbf{X}(k)$ is linear in $\mathbf{S}(k)$ but nonlinear in $\Theta$.
A standard procedure of solving this type of joint maximization problem which contains both linear and nonlinear parameters is as follows. (1) Fix the nonlinear parameter and derive the optimal estimator of the linear parameter. (2) Substitute the linear parameter by its estimator in the original objective function and obtain a concentrated objective function that contains only the nonlinear parameter. (3) Find the estimate for the nonlinear parameter by optimizing the concentrated objective function. (4) Find the estimate for the linear parameter by substituting the estimate for the nonlinear parameter back to its estimator. Follow this procedure, the original joint optimization problem is reduced to a separable optimization problem.

For our wideband DOA estimation problem (9), once $\Theta$ is fixed, the estimator for $\mathbf{S}$ is simply the least-squares solution

$$
\widehat{\mathbf{S}}(k)=\mathbf{D}(k)^{\dagger} \mathbf{X}(k)
$$

Substituting $\widehat{\mathbf{S}}(k)$ back to (9) and the estimator for $\boldsymbol{\Theta}$ can then be written as

$$
\widehat{\boldsymbol{\Theta}}=\arg \min _{\boldsymbol{\Theta}} \sum_{k=1}^{N / 2}\left\|\mathbf{X}(k)-\mathbf{D}(k)^{\dagger} \mathbf{X}(k)\right\|^{2} .
$$

Note that we start with a joint optimization problem (8) of dimension $M+P N+1$, and then reduce it analytically to a much smaller optimization problem (11) of dimension $M$. Many numerical optimization algorithms in the literature can be used to solve $\widehat{\boldsymbol{\Theta}}[20-26]$. No methods are guaranteed to achieve the global optimum in general.

\section{DERIVATION OF THE WIDEBAND MAXIMUM-LIKELIHOOD DOA ESTIMATOR AND THE CRB IN THE PRESENCE OF NONUNIFORM SENSOR NOISE}

In this section, we derive a new nonuniform wideband DOA ML estimator and the CRB in the presence of nonuniform sensor noise. Unlike the uniform white noise model used in Section 3, the noise covariance matrix $\mathbf{Q}$ is now modeled as a diagonal matrix with nonidentical diagonal elements (3).

Define $\boldsymbol{\Psi}=\left[\boldsymbol{\Theta}^{T}, \mathbf{S}^{T}, \mathbf{q}^{T}\right]^{T}$ as the vector of all the unknown parameters in the model, where $\mathbf{q}=\left[q_{1}, \ldots, q_{P}\right]^{T}$ is the vector of the diagonal elements of $\mathbf{Q}$, then the likelihood function of $\Psi$ can be expressed as

$$
f(\Psi)=\frac{1}{\left\{\pi^{P} \operatorname{det} \mathbf{Q}\right\}^{N / 2}} \exp \left\{-\sum_{k=1}^{N / 2} \mathbf{g}(k)^{H} \mathbf{Q}^{-1} \mathbf{g}(k)\right\} .
$$

Taking the logarithm of (12) and neglecting all the constant terms, we have the following log-likelihood function $L(\Psi)$ :

$$
L(\Psi)=-\frac{N}{2} \sum_{p=1}^{P} \log q_{p}-\sum_{k=1}^{N / 2}\|\tilde{\mathbf{g}}(k)\|^{2},
$$

where

$$
\begin{aligned}
\tilde{\mathbf{g}}(k) & =\mathbf{Q}^{-1 / 2} \mathbf{g}(k)=\tilde{\mathbf{X}}(k)-\tilde{\mathbf{D}}(k) \mathbf{S}(k), \\
\tilde{\mathbf{X}}(k) & =\mathbf{Q}^{-1 / 2} \mathbf{X}(k), \\
\widetilde{\mathbf{D}}(k) & =\mathbf{Q}^{-1 / 2} \mathbf{D}(k) .
\end{aligned}
$$


$\tilde{\mathbf{g}}(k), \tilde{\mathbf{X}}(k)$, and $\tilde{\mathbf{D}}(k)$ can be viewed as the "spatially whitened" version of $\mathbf{g}(k), \mathbf{X}(k)$, and $\mathbf{D}(k)$, respectively.

It follows that the maximum likelihood estimator for $\Psi$ is simply

$$
\hat{\Psi}=\arg \max _{\Psi} L(\Psi),
$$

which is a joint optimization problem of dimension $M+$ $M N+P$. Since the estimation of $\Theta$ is our only interest, we would like to reduce the search space analytically as is done in the derivation of the uniform-ML estimator.

Unlike the uniform noise case, now the estimation of $\boldsymbol{\Theta}$ and $\mathbf{S}$ is through $\|\widetilde{\mathbf{g}}(k)\|^{2}$, which is also a function of $\mathbf{q}$. Therefore, the estimation of signal parameters and noise parameters are interrelated. We approach this problem by first fixing $\Theta$ and $\mathbf{S}$ in (15) and deriving an estimator of $\mathbf{q}$ as a function of $\Theta$ and $\mathbf{S}$. After taking the gradient of $L(\Psi)$ with respect to $\mathbf{q}$ and setting it to zero, the following estimator for $q_{p}$ is obtained:

$$
\begin{aligned}
\hat{q}_{p} & =\frac{2}{N} \sum_{k=1}^{N / 2}\left|[\mathbf{g}(k)]_{p}\right|^{2} \\
& =\frac{2}{N}\left\|\mathbf{g}_{p}\right\|^{2}
\end{aligned}
$$

where $[\mathbf{g}(k)]_{p}$ denotes the $p$ th element of the residual vector, $\mathbf{g}(k)$, and

$$
\mathbf{g}_{p}=\left[[\mathbf{g}(1)]_{p}, \ldots,\left[\mathbf{g}\left(\frac{N}{2}\right)\right]_{p}\right]^{T}
$$

Let $\hat{\mathbf{q}}=\left[\hat{q}_{1}, \ldots, \hat{q}_{P}\right]^{T}$ and substitute $\mathbf{q}$ by $\hat{\mathbf{q}}$ in (13), we have the following concentrated log-likelihood function of $\boldsymbol{\Theta}$ and $\mathbf{S}$ :

$$
\begin{aligned}
L(\boldsymbol{\Theta}, \mathbf{S}) & =-\frac{N}{2} \sum_{p=1}^{P} \log \hat{q}_{p}-\sum_{k=1}^{N / 2} \sum_{p=1}^{P} \frac{\left|[\mathbf{g}(k)]_{p}\right|^{2}}{\hat{q}_{p}} \\
& =\frac{N}{2}\left\{P\left[\log \left(\frac{N}{2}\right)-1\right]-\sum_{p=1}^{P} \log \left\|\mathbf{g}_{p}\right\|^{2}\right\} .
\end{aligned}
$$

It follows that a concentrated ML estimator for $\boldsymbol{\Theta}$ and $\mathbf{S}$ can be expressed as

$$
(\hat{\boldsymbol{\Theta}}, \widehat{\mathbf{S}})=\arg \max _{\boldsymbol{\Theta}, \mathbf{S}}-\sum_{p=1}^{P} \log \left\|\mathbf{g}_{p}\right\|^{2} .
$$

To concentrate $L(\boldsymbol{\Theta}, \mathbf{S})$ further, one would fix $\boldsymbol{\Theta}$ and derive an estimator for $\mathbf{S}$ that maximizes $L(\boldsymbol{\Theta}, \mathbf{S})$. Unfortunately, a separable closed-form estimator for $\boldsymbol{S}$ that maximizes (20) seems to be analytically unavailable, and this prevents us from further simplifications.

On the other hand, we can approach the problem by fixing $\Theta$ and $\mathbf{q}$ in (15) and derive an estimator for $\mathbf{S}$. The resulting estimator $\widehat{\mathbf{S}}$ is again the least-square solution

$$
\widehat{\mathbf{S}}(k)=\widetilde{\mathbf{D}}(k)^{\dagger} \tilde{\mathbf{X}}(k) .
$$

Substituting $\mathbf{S}(k)$ by $\widehat{\mathbf{S}}(k)$ into (13), the concentrated loglikelihood function of $\boldsymbol{\Theta}$ and $\mathbf{Q}$ is obtained as

$$
L(\boldsymbol{\Theta}, \mathbf{Q})=-\frac{N}{2} \sum_{p=1}^{P} \log q_{p}-\sum_{k=1}^{N / 2}\left\|\mathbf{P}_{\tilde{\mathbf{D}}(\mathbf{k})}^{\perp} \tilde{\mathbf{X}}(k)\right\|^{2},
$$

where

$$
\mathbf{P}_{\tilde{\mathbf{D}}(\mathbf{k})}^{\perp}=\mathbf{I}-\tilde{\mathbf{D}}(k) \widetilde{\mathbf{D}}(k)^{\dagger} .
$$

As a result, a concentrated $\mathrm{ML}$ estimator for $\boldsymbol{\Theta}$ and $\mathbf{Q}$ is obtained as

$$
(\widehat{\boldsymbol{\Theta}}, \widehat{\mathbf{Q}})=\arg \max _{\boldsymbol{\Theta}, \mathbf{Q}} L(\boldsymbol{\Theta}, \mathbf{Q}) .
$$

Again, no closed-form estimator of $\mathbf{Q}$ which maximizes (22) with fixed $\Theta$ seems to be available, and therefore no further concentration can be performed. Instead of direct implementing (15), (20), or (24), which requires an exhaustive search in the joint parameter space, we propose the following two novel algorithms to reduce the complexity.

\subsection{Stepwise-concentrated maximum likelihood algorithm (SC-ML)}

The first proposed algorithm is based on the technique of stepwise concentration (SC), which is conceptually related to the alternating projection (AP) [2], iterative quadratic maximum likelihood (IQML) [27], and method of direction estimation (MODE) [28]. The idea of this technique is to stepwise concentrate the log-likelihood function in an iterative manner, which has been successfully applied in [18]. In this subsection, we use the same concept to numerically concentrate (20).

Insert (21) into (20), we have the following alternative expression:

$$
(\widehat{\boldsymbol{\Theta}}, \hat{\mathbf{Q}})=\arg \max _{\boldsymbol{\Theta}, \mathbf{Q}} \bar{L}(\boldsymbol{\Theta}, \mathbf{Q}),
$$

where

$$
\begin{gathered}
\bar{L}(\boldsymbol{\Theta}, \mathbf{Q})=-\sum_{p=1}^{P} \log \left\|\overline{\mathbf{g}}_{p}\right\|^{2}, \\
\overline{\mathbf{g}}_{p}=[[\overline{\mathbf{g}}(1)]]_{p}, \ldots,\left[\overline{\mathbf{g}}\left(\frac{N}{2}\right)_{p}\right]^{T}, \\
\overline{\mathbf{g}}(k)=\mathbf{X}(k)-\mathbf{D}(k) \widetilde{\mathbf{D}}(k)^{\dagger} \tilde{\mathbf{X}}(k) .
\end{gathered}
$$

Using (17), (21), and (25), we have the iterative procedure shown in Algorithm 1.

In the proposed SC-ML, we initialize the procedure by assuming the noise covariance matrix $\hat{\mathbf{Q}}=\mathbf{I}$. In fact, this initialization is less restrictive than it appears. For a more general noise covariance matrix $\hat{\mathbf{Q}}=\alpha \mathbf{I}$, where $\alpha$ is an arbitrary positive constant, it is easy to show that for a fixed $\alpha$, the first term of (22) is simply a constant while the second term is not a function of $\alpha$. As a result, the DOA estimate obtained at step 1 is independent of the value of $\alpha$, which allows us to 
Initialization: Iter $=0$. Set $\hat{\mathbf{Q}}=\mathbf{I}($ same as setting $\hat{\mathbf{q}}=\mathbf{1})$.

Loop start:

Step 1. Find the estimate of $\Theta$ as $\widehat{\boldsymbol{\Theta}}=\arg \max _{\Theta} \bar{L}(\boldsymbol{\Theta}, \widehat{\mathbf{Q}})$, where $\bar{L}(\boldsymbol{\Theta}, \mathbf{Q})$ is defined as in $(26)-(28)$. Iter $=$ Iter +1 .

Step 2. Use the obtained $\widehat{\boldsymbol{\Theta}}$ and $\hat{\mathbf{q}}$ to compute $\widehat{\mathbf{S}}(k)$ through $(21)$.

Step 3. Use the obtained $\widehat{\boldsymbol{\Theta}}$ and $\widehat{\mathbf{S}}(k)$ to compute a refined $\hat{\mathbf{q}}$ through (17).

Loop end:

Repeat steps $1-3$ a few times to obtain the final estimate.

Algorithm 1: Iterative procedure of the proposed SC-ML algorithm.

simply set $\alpha=1(\hat{\mathbf{Q}}=\mathbf{I})$ for a more general uniform noise initialization. The convergence of the algorithm follows from the fact that a new set of parameters is found in each iteration such that $\bar{L}(\widehat{\boldsymbol{\Theta}}, \widehat{\mathbf{Q}})$ is monotonically increasing. This ensures that the algorithm converges to at least a local optimal point. Simulation results (Section 5) also show that only two iterations are required to obtain a solution close to the CRB.

It is also interesting to observe that the concentrated $\log$ likelihood function (26) used in the SC-ML in the uniform case does not degenerate to the log-likelihood used in the uniform-ML estimator (11). This is because when we substitute (17) into (13), the a priori information on the structure of the noise covariance matrix has been exploited explicitly. This prior information is processed through the logarithmic operation which serves as an "equalizer" assigning lower weighting to noisier sensors.

Like the uniform-ML estimator, the major computational burden of the SC-ML is in the DOA estimation stage of step 1 , where a highly nonlinear optimization problem needs to be solved. Many numerical algorithms designed for solving (11) in the literature can be easily modified to carry out step 1.

\subsection{Approximately concentrated maximum likelihood algorithm (AC-ML)}

In Section 4.1, an iterative maximum likelihood wideband DOA estimation algorithm is presented. The algorithm stepwise concentrates the DOAs and the nuisance parameters, and it only requires a few iterations to converge to a solution close to the CRB (see Section 5). In this subsection, we propose a new algorithm which is noniterative and has an MSE performance comparable to the SC-ML.

Clearly, a naive noniterative algorithm is already available, which can be obtained by simply running the SC-ML for just one single iteration without any refinement. Therefore, for the proposed noniterative algorithm to be useful, it must give a consistently better performance in comparison to the 1st-iteration estimate of the SC-ML. In Section 4.1, we describe the procedure of the SC-ML, which initializes the algorithm by setting $\widehat{\mathbf{Q}}=\mathbf{I}$. Intuitively, a better estimator can be developed if the algorithm starts with a more informative Q.

This is the main idea of our AC-ML. Instead of initializing the algorithm with a constant $\hat{\mathbf{q}}=\mathbf{1}$, we seek a $\breve{\mathbf{q}}(\boldsymbol{\Theta})$ so that as we optimize over $\boldsymbol{\Theta}$, we optimize over $\check{\mathbf{q}}$ simultaneously. For the AC-ML, we propose the following $\check{\text { q̀: }}$

$$
\check{\mathbf{q}}=\left[\check{q}_{1}, \ldots, \check{q}_{P}\right]^{T},
$$

where

$$
\begin{gathered}
\check{q}_{p}=\frac{2}{N}\left\|\left[[\check{\mathbf{g}}(1)]_{p}, \ldots,\left[\check{\mathrm{g}}\left(\frac{N}{2}\right)\right]_{p}\right]^{T}\right\|^{2}, \\
\check{\mathbf{g}}(k)=\mathbf{X}(k)-\mathbf{D}(k) \mathbf{D}(k)^{\dagger} \mathbf{X}(k) .
\end{gathered}
$$

The proposed $\mathbf{q}$ has the same expression as $\hat{\mathbf{q}}$ in (17), except the $\mathbf{S}(k)$ is now approximated by $\mathbf{D}(k)^{\dagger} \mathbf{X}(k)$. Recall $\mathbf{D}(k)^{\dagger} \mathbf{X}(k)$ is the ML estimator for $\mathbf{S}(k)$ under the white noise assumption. At high SNR region, $\mathbf{D}(k)^{\dagger} \mathbf{X}(k)$ appears to be a good approximation for $\mathbf{S}(k)$ and as a consequence q̌ provides a nice estimate of the underlying nonuniform noise. With this approximation, we now have the proposed AC-ML:

$$
\widehat{\boldsymbol{\Theta}}=\arg \min _{\boldsymbol{\Theta}} \sum_{p=1}^{P} \log \left\|\widehat{\mathbf{g}}_{p}\right\|^{2}
$$

where

$$
\begin{gathered}
\hat{\mathbf{g}}_{p}=\left[[\hat{\mathbf{g}}(1)]_{p}, \ldots,\left[\hat{\mathbf{g}}\left(\frac{N}{2}\right)\right]_{p}\right]^{T}, \\
\hat{\mathbf{g}}(k)=\mathbf{X}(k)-\mathbf{D}(k) \check{\mathbf{D}}(k)^{\dagger} \check{\mathbf{X}}(k), \\
\check{\mathbf{D}}(k)=\check{\mathbf{Q}}^{-1 / 2} \mathbf{D}(k), \\
\check{\mathbf{X}}(k)=\check{\mathbf{Q}}^{-1 / 2} \mathbf{X}(k), \\
\check{\mathbf{Q}}=\operatorname{diag}\{\check{\mathbf{q}}\} .
\end{gathered}
$$

Strictly speaking, the AC-ML is a suboptimal algorithm and can be made an iterative algorithm by the same stepwiseconcentration technique. However, it is shown in the simulations of Section 5 that the AC-ML gives almost the same performance of the SC-ML within the SNR regions of interest and provides a solution close to the derived CRB high SNR, which suggests no significant MSE performance can be gained through iterative refinement.

The complexity of the AC-ML is again dominated by the DOA estimation stage (32), where a global optimization problem needs to be solved. To be more specific, the complexity is dominated by the pseudoinverse operation in (31) 
and (34), and the logarithmic function evaluation in (32). For the SC-ML, one pseudoinverse operation in (28) and one logarithmic function evaluation are required for every testing $\Theta$ in each iteration. A more detailed complexity comparison between the SC-ML and AC-ML depends on the choice of optimization methods. Nonetheless, if we assume the same optimization algorithm for both estimators and the efforts of each iteration in the SC-ML are roughly the same, we can conclude the complexity of the AC-ML is less than that of the $\mathrm{SC}-\mathrm{ML}$ running two iterations.

\subsection{The Cramér-Rao bound}

The CRB is probably the most well-known theoretical bound of the variances of unbiased estimators. In this subsection, we present the results of the CRB derived from the wideband signal and nonuniform sensor noise model (Section 2). The newly derived nonuniform CRB can be viewed as an extension of [29] to a more general multiple sources/nonuniform noise scenario and the wideband generalization of the narrowband deterministic expression shown in [18]. The detailed derivation is shown in Appendix A.

Lemma 1. The inverse of the CRB matrix for $\Theta$ can be expressed as

$$
\mathbf{C R B}_{\Theta \Theta}^{-1}=2 \Re\left\{\sum_{k=1}^{N / 2}\left\{\left[\widetilde{\mathbf{E}}(k)^{H} \mathbf{P}_{\widetilde{\mathbf{D}}(\mathbf{k})}^{\perp} \widetilde{\mathbf{E}}(k)\right] \odot \mathbf{R}_{\mathbf{S}}(k)^{T}\right\}\right\},
$$

where

$$
\begin{aligned}
\mathbf{P}_{\tilde{\mathbf{D}}(\mathbf{k})}^{\perp} & =\mathbf{I}-\widetilde{\mathbf{D}}(\mathbf{k}) \widetilde{\mathbf{D}}(\mathbf{k})^{\dagger}, \\
\widetilde{\mathbf{E}}(k) & =\mathbf{Q}^{-1 / 2} \mathbf{E}(k), \\
\mathbf{E}(k) & =\left[\frac{d}{d \theta_{1}} \mathbf{d}^{(1)}(k), \ldots, \frac{d}{d \theta_{M}} \mathbf{d}^{(M)}(k)\right], \\
\mathbf{R}_{\mathbf{S}}(k) & =\mathbf{S}(k) \mathbf{S}(k)^{H} .
\end{aligned}
$$

When the sensor noise is uniform, (36) degenerates to

$$
\mathbf{C R B}_{\boldsymbol{\Theta} \Theta \text {,uni }}^{-1}=\frac{2}{\sigma^{2}} \mathfrak{R}\left\{\sum_{k=1}^{N / 2}\left\{\left[\mathbf{E}(k)^{H} \mathbf{P}_{\mathbf{D}(k)}^{\perp} \mathbf{E}(k)\right] \odot \mathbf{R}_{\mathbf{S}}(k)^{T}\right\}\right\} .
$$

From (36) we observe that $\mathbf{C R B}_{\Theta \Theta}^{-1}$ contains contributions from all frequency bins through a direct summation. The contribution from each frequency bin is an elementwise matrix product of the geometry factor, $\widetilde{\mathbf{E}}(k)^{H} \mathbf{P}_{\widetilde{\mathbf{D}}(\mathbf{k})}^{\perp} \widetilde{\mathbf{E}}(k)$, and the spectral factor, $\mathbf{R}_{\mathbf{S}}(k)^{T}$. The geometry factor provides a measure of geometric relations between the sources and the sensor array, and the significance of each sensor is adjusted by its variance through $\widetilde{\mathbf{D}}(k)$ and $\widetilde{\mathbf{E}}(k) \cdot \mathbf{Q}^{-1 / 2}$ acts as the manifold transformation matrix which spatially prewhitens $\mathbf{D}(k)$ and $\mathbf{E}(k)$ so that noisy sensors are given less weights. The spectral factor $\mathbf{R}_{\boldsymbol{S}}(k)^{T}$, on the other hand, provides a measure of correlations among $M$ sources. For those frequency bins where no signals are present, the spectral factors are just zero matrices and thus do not contribute to $\mathbf{C R B}_{\Theta \Theta}^{-1}$ as is intuitively expected.
Under the single source scenario, (36) can be further simplified as

$$
\mathbf{C R B}^{-1}=2 \sum_{k=1}^{N / 2}\left\{\|\tilde{\mathbf{e}}(k)\|^{2}\|\tilde{\mathbf{d}}(k)\|^{2}-\left|\tilde{\mathbf{d}}(k)^{H} \tilde{\mathbf{e}}(k)\right|^{2}\right\} \frac{|S(k)|^{2}}{\|\tilde{\mathbf{d}}(k)\|^{2}} .
$$

Here we have changed the notation $\mathbf{S}(k)$ to $S(k)$, since $S(k)$ is just a complex scalar in the single source scenario. Generalizing the results of $[18,30]$ we can define the wideband array-signal-to-noise ratio (ASNR):

$$
\operatorname{ASNR}=\frac{2}{N P} \sum_{k=1}^{N / 2}\|\tilde{\mathbf{d}}(k)\|^{2}|S(k)|^{2},
$$

which is a measure of the averaged SNR. If we further assume the sensors to be omnidirectional with unit sensor response $\left(a_{p}(k, \theta)=1\right)$ and have the same noise variance $\sigma^{2},(40)$ becomes

$$
\operatorname{ASNR}=\frac{2}{N \sigma^{2}} \sum_{k=1}^{N / 2}|S(k)|^{2}
$$

which is the same as the common definition for the SNR. This quantity (40) will be fixed when we investigate the effect of the nonuniformity of noise in Section 5.

\section{SIMULATIONS}

In this section, we present the simulation results of the proposed algorithms under varies of simulation examples. While the assumed signal models and the proposed algorithms are derived for general wideband applications, the simulation examples demonstrated in this section will be performed under the acoustic settings.

The simulation is performed under a setup of an 8element UCA with a radius $R=0.25$ meter. Each microphone on the UCA is assumed to be omnidirectional with unity sensor gain $\left(a_{p}\left(k, \theta_{m}\right)=1\right)$ and perfectly synchronized. Three human speech recordings sampled at $16 \mathrm{kHz}$ are used as our wideband sources (Figure 1), and a frame of 1024 snapshots (64 milliseconds) has been extracted from each recording (see Figures 2 and 3 ). The propagation speed of acoustic wave is set to be $345 \mathrm{~m} / \mathrm{s}$ and is assumed to be known.

Throughout the simulation, the DOA estimation operations required in step 1 of the SC-ML and (32) of the AC-ML are performed by the alternating maximization (AM) algorithm [31], implemented by a coarse search of $1^{\circ}$ step size followed by the golden section fine search. Theoretical results as well as the relative capabilities of the proposed algorithms (SC-ML and AC-ML) are shown in the following examples.

\section{(1) The wideband CRB in the presence of nonuniform sensor noise}

Unlike most simulation settings presented in the literature, we choose a UCA rather than a uniform linear array (ULA) 


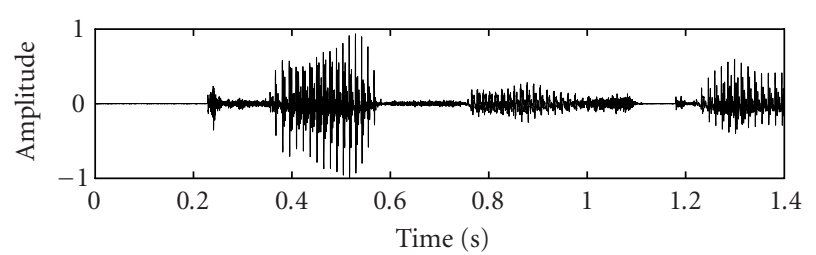

(a)

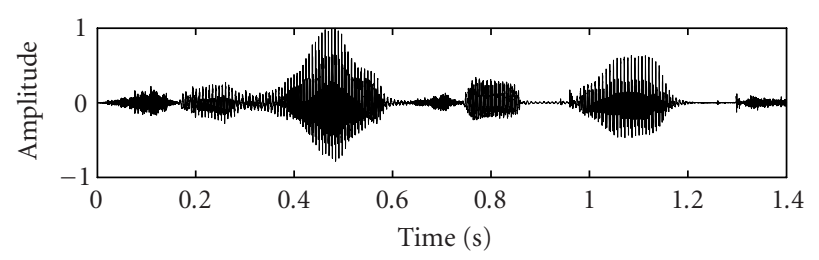

(b)

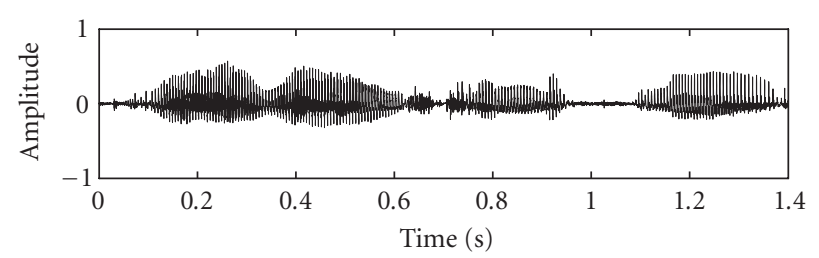

(c)

Figure 1: Acoustic waveforms of three human speech recordings.

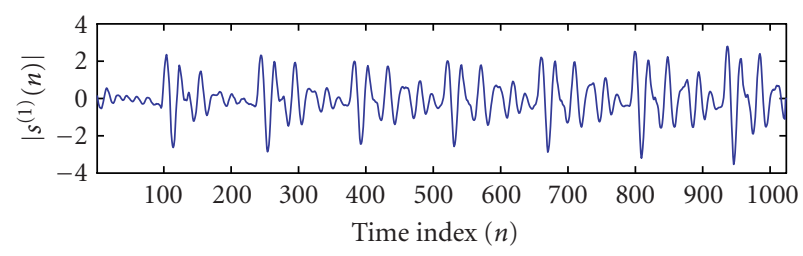

(a)

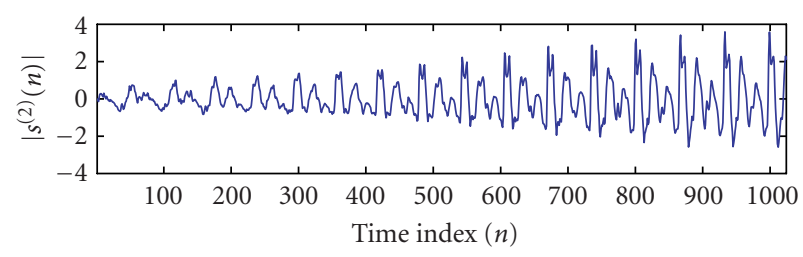

(b)

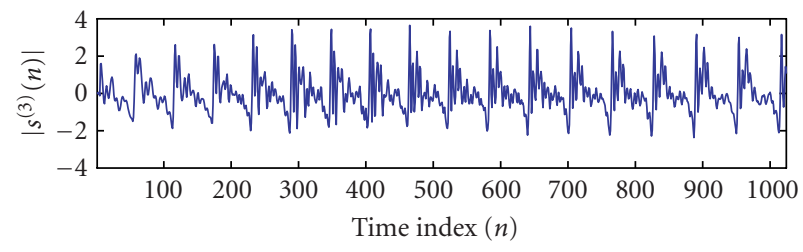

(c)

FIGURE 2: Time domain acoustic waveforms of the extracted frame (after normalization).

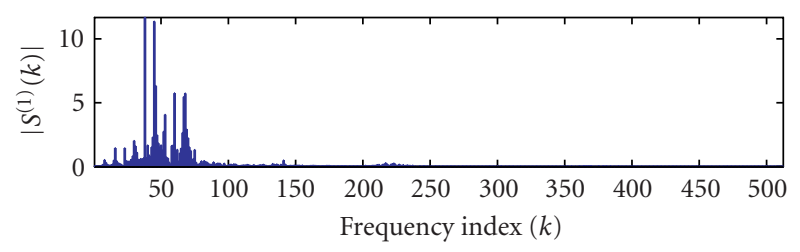

(a)

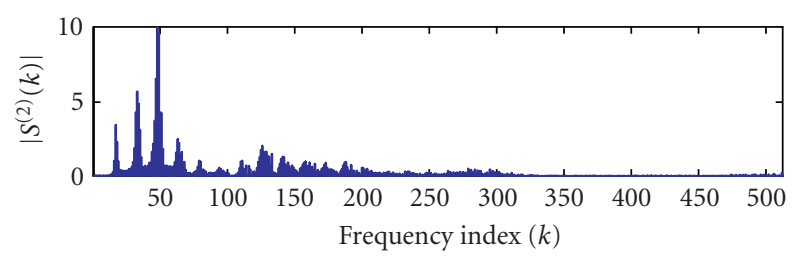

(b)

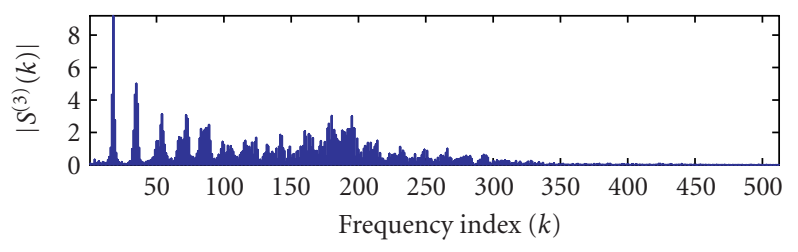

(c)

Figure 3: Magnitude spectrum of the extracted frame (after normalization).

as our array geometry. Although the ULA is easy to analyze and provides the largest array aperture when given the same number of sensors, it has a few restrictions. First, the ULA is unable to distinguish DOAs symmetric to the array line and therefore is usually applied to applications where the field of view (FOV) is within $180^{\circ}$ [32]. Second, the performance of the ULA is nonuniform. For example, the DOA estimation performance degrades considerably near the endfire of a ULA, while the UCA always gives uniform performance over the whole FOV for single source DOA estimation $[29,33]$. As a result, the UCA has been considered as one of the most favorable geometries used in DOA estimation. However, this nice property holds only under the uniform white noise assumption. When the sensor noise is nonuniform, the CRB becomes a function of the DOAs and the dependency varies with the distribution of the noise variances.

In the first example, we investigate how the nonuniformity of the noise affects the theoretical capabilities of the DOA estimation. Source 1 is chosen as our wideband source in this example and DOA1 is assumed to be $90^{\circ}$ relative to the array. Let us define the worst-noise-power ratio (WNPR) [18]:

$$
\mathrm{WNPR}=\frac{\sigma_{\max }^{2}}{\sigma_{\min }^{2}}
$$

as a measurement of nonuniformity of sensor noise, where $\sigma_{\max }^{2}$ and $\sigma_{\min }^{2}$ are the largest and smallest noise variance, 


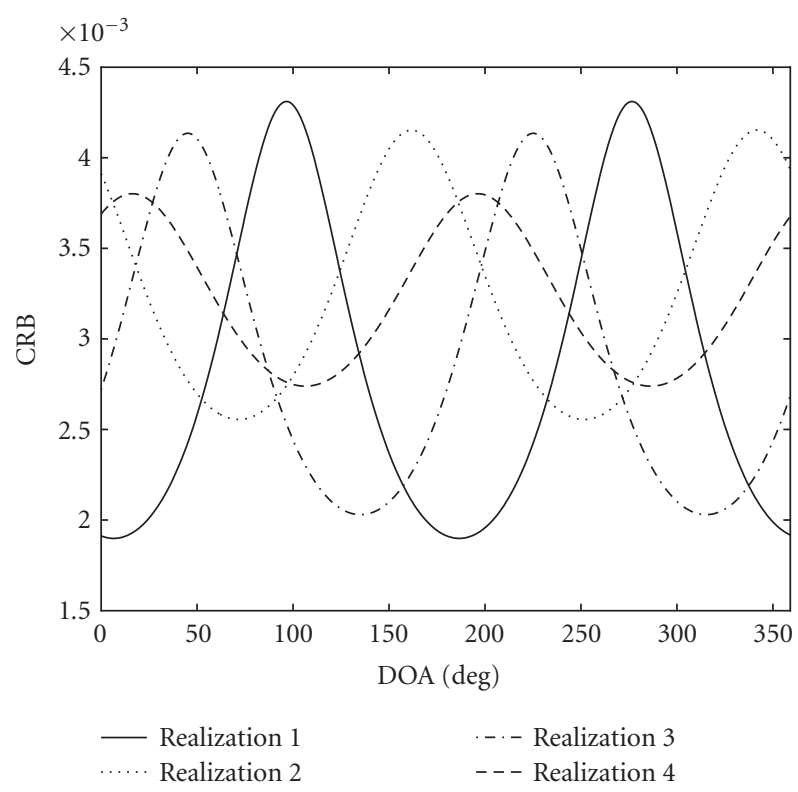

FIgURe 4: CRB versus the source DOA.

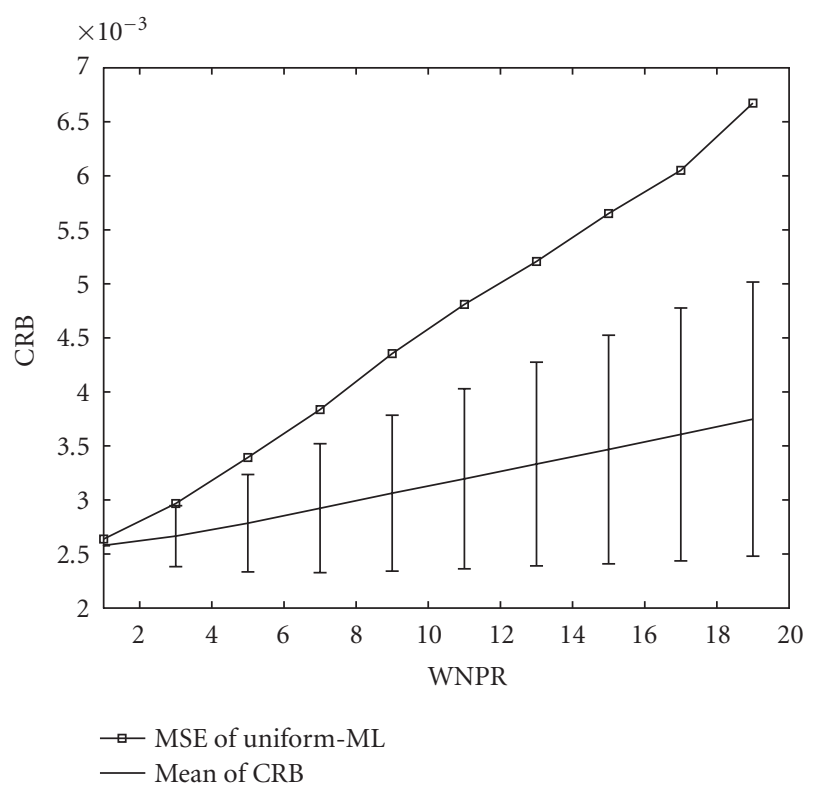

FIGURE 5: Comparison of the MSE of the uniform-ML estimator with the CRB.

respectively. In each Monte Carlo run, we fix the WNPR and randomly choose two sensor locations in the UCA, one with noise variance $\sigma_{\min }^{2}$ and the other $\sigma_{\max }^{2}=\mathrm{WNPR} \sigma_{\min }^{2}$. The noise variance of the rest of the sensors are assigned according to a uniform distribution within the interval $\left(\sigma_{\min }^{2}, \sigma_{\max }^{2}\right)$. In order to reduce the effect of SNR fluctuations, the noise variance is scaled such that the ASNR defined in (40) is fixed at $20 \mathrm{~dB}$.

In Figure 4, we fix the WNPR to be 20 and perform the simulation described as before. The CRBs from four random realizations in the Monte Carlo runs have been plotted with

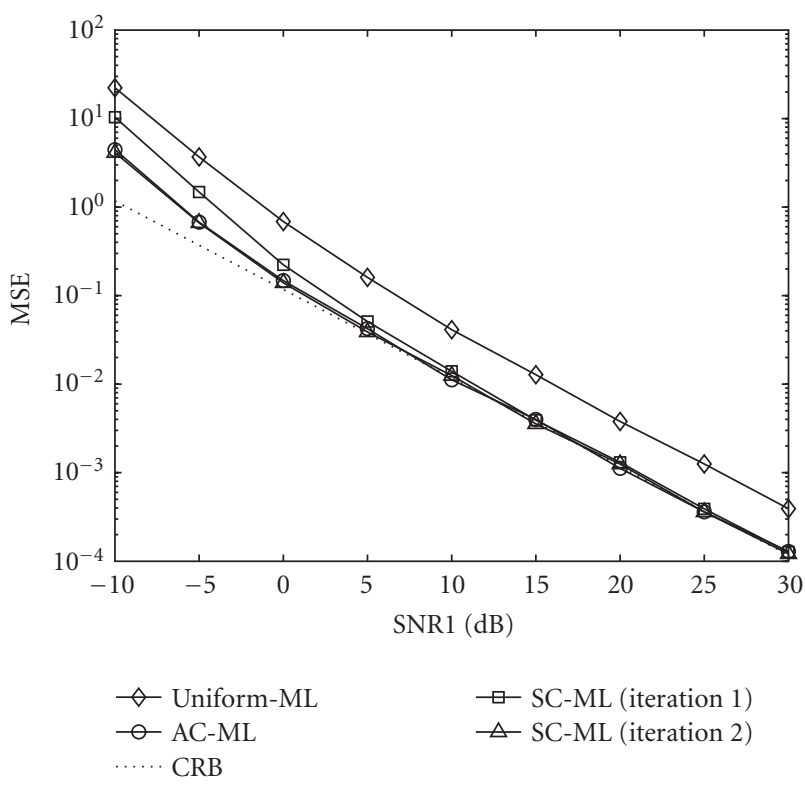

FIgure 6: Comparison of the DOA estimation MSEs and the CRB (single source case).

respect to the DOA. It is clear that the CRB of a UCA in the presence of nonuniform noise is no longer a constant over the FOV and depends on the nonuniformity of noise. It is also observed that the nonuniform CRB has a period of $180^{\circ}$. This is true for any nonisotropic planar array, which can be easily verified from (39) (see Appendix B).

Figure 5 further quantifies the relation of the nonuniformity of the CRB with respect to the WNPR. The mean and standard deviation (std) of the CRB shown on the error bar of the figure is estimated by averaging over 10000 Monte Carlo simulations. As expected, the CRB has a larger std as the WNPR increases and therefore is more nonuniform. We also plot the MSE of the uniform-ML estimator [12] under the same simulation conditions. The vertical gap between the MSE of the uniform-ML estimator and the mean of CRB shows the average performance degradation if the nonuniformity of noise is ignored. A larger average performance degradation is also observed at high WNPR. This justifies the development of the SC-ML and AC-ML presented in this work. When the noise is uniform (WNPR = 1), the performance loss between the uniform-ML estimator and the CRB is zero.

\section{(2) Single source wideband DOA estimation}

In the second example, we investigate the performance of the SC-ML and the AC-ML in estimating the DOA of a single source in the presence of nonuniform sensor noise. The setting of the simulation is the same as the previous example except now the covariance of the sensor noise is fixed to

$$
\mathbf{Q}=\sigma^{2} \operatorname{diag}\{1,20,1.5,1,10,1,2,5\} .
$$

The MSEs and the CRB are plotted with respect to the SNR of the 1st sensor for comparison. 


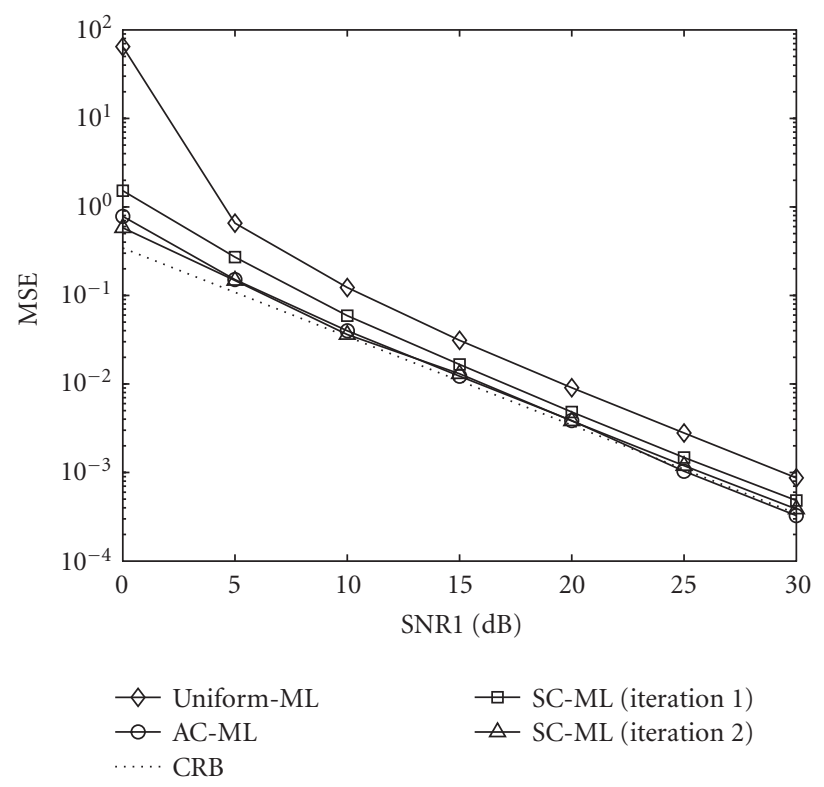

FIgure 7: Comparison of the MSEs and the CRB in estimating DOA1 (two sources case).

Figure 6 shows the MSE performance of the SC-ML, AC$\mathrm{ML}$, and the conventional uniform-ML estimator with respect to the SNR. Each simulation point on the figure is computed by averaging over 500 Monte Carlo runs. The SC-ML converges quickly within two iterations within the SNR of interest, and even converges in 1 iteration when SNR is higher than $10 \mathrm{~dB}$. The AC-ML, on the other hand, gives almost the same performance of the SC-ML within the SNR of interest. Both algorithms achieve the derived CRB at high SNR while the conventional uniform-ML estimator does not. At low SNR, the CRB is not attained. This is due to the threshold effect caused by the occasionally occurred outliers and is a common phenomenon for nonlinear estimators [34].

\section{(3) Two sources wideband DOA estimation}

In the third example, two wideband sources (source 1 and source 2 in Figures 2 and 3 ) are assumed where DOA1 and DOA2 are set to be at $90^{\circ}$ and $120^{\circ}$, respectively. The received waveforms from two sources overlap both in time and frequency and are normalized to have equal averaged power. The noise covariance is again fixed to (43), and the MSEs and the CRB are plotted with respect to the SNR of the 1st sensor.

Figures 7 and 8 show the the MSEs and CRBs of DOA1 and DOA2, respectively. Again the SC-ML is able to obtain a solution close to the CRB within two iterations at a high SNR. The AC-ML on contrary achieves almost comparable MSE performance as the SC-ML and is consistently better than both the 1 iteration estimate of the SC-ML and the uniformML estimator.

\section{(4) Three sources wideband DOA estimation}

In the fourth example, we fix the noise covariance matrix to (43) as in examples 2 and 3, but the number of sources

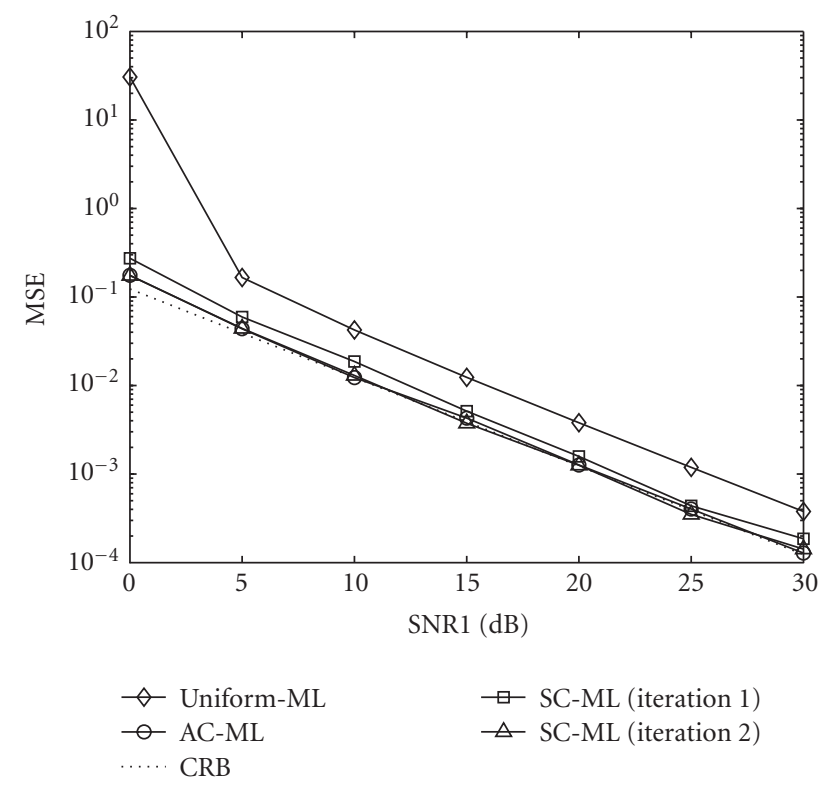

FIGURE 8: Comparison of the MSEs and the CRB in estimating DOA2 (two sources case).

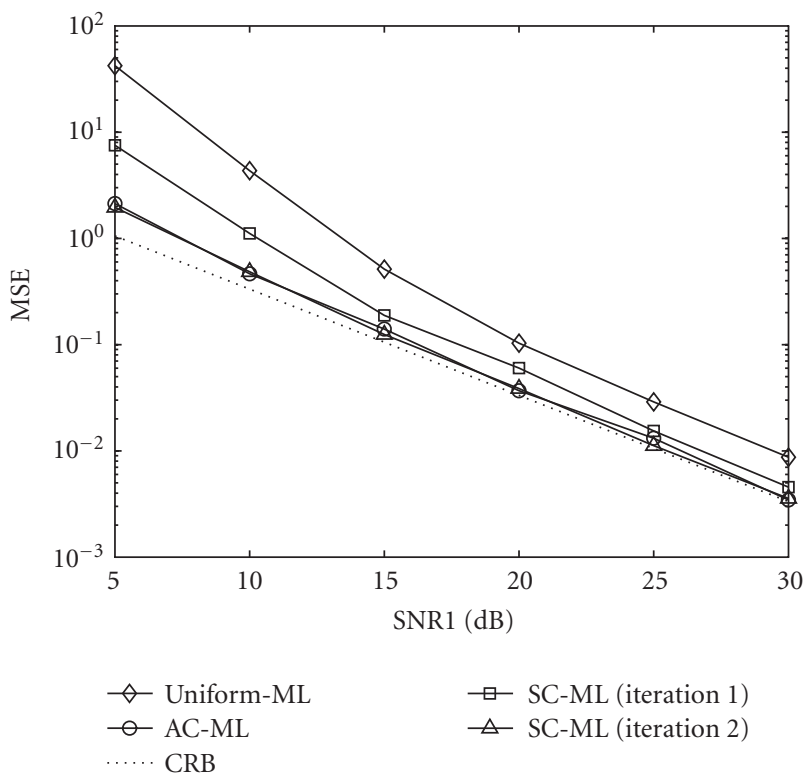

FIgure 9: Comparison of the MSEs and the CRB in estimating DOA1 (three sources case).

(normalized to have equal power) is now increased to three with the following DOAs $\left(75^{\circ}, 105^{\circ}\right.$, and $135^{\circ}$, resp.). The corresponding MSEs and the CRBs for DOA1 to DOA3 are shown in Figures 9-11 plotted with respect to the SNR of the 1st sensor. Similar observations can be made for the three sources case. The AC-ML consistently outperforms the 1st iteration estimate of the SC-ML and both the AC-ML and SC-ML (running only 2 iterations) obtain a solution close to the CRB at a high SNR. 


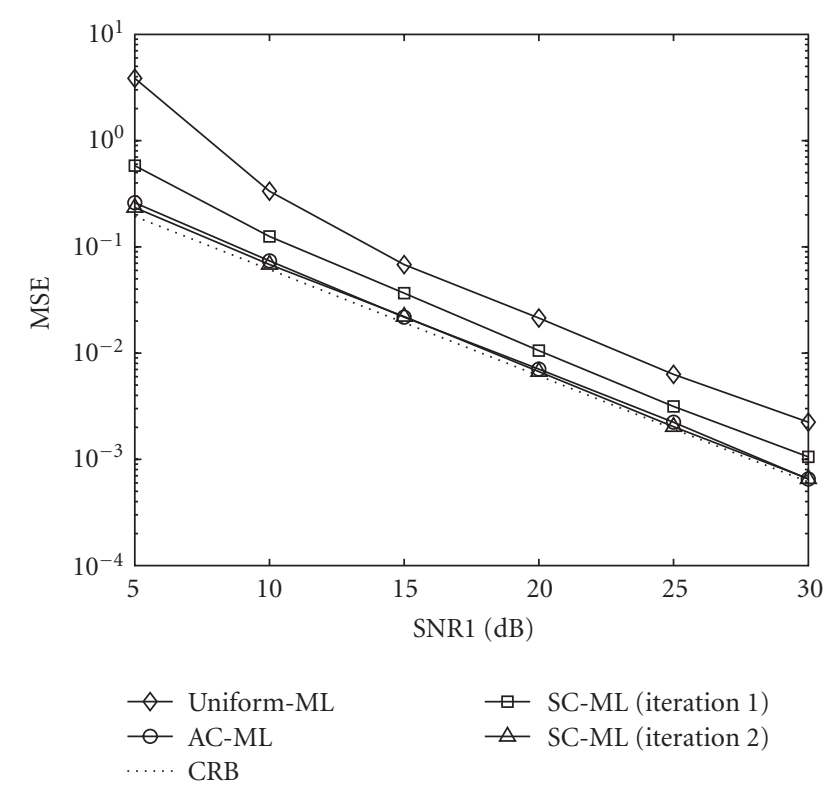

FIgUre 10: Comparison of the MSEs and the CRB in estimating DOA2 (three sources case).

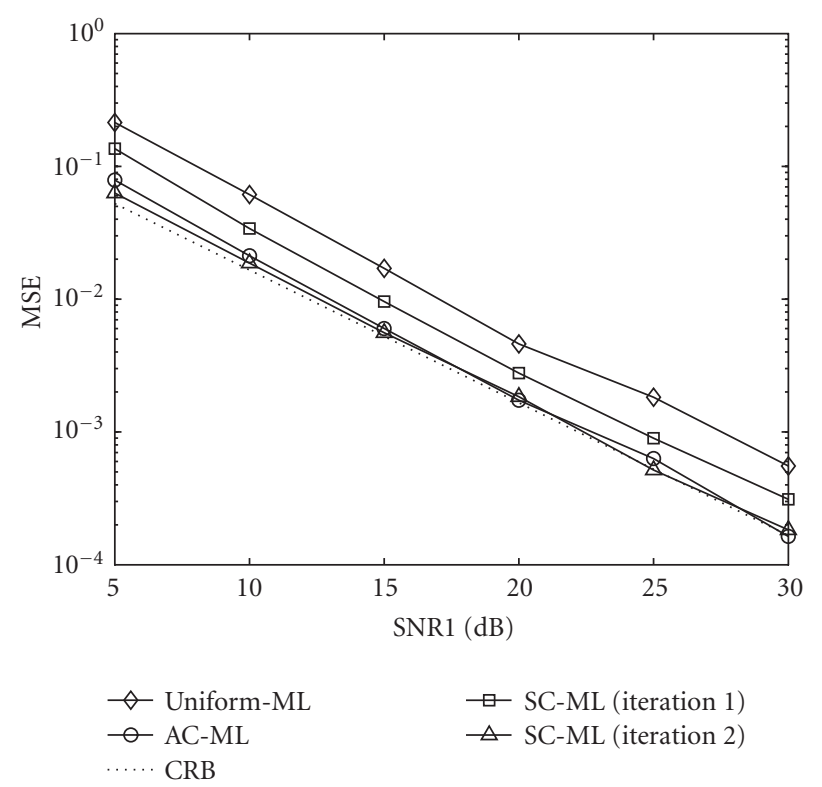

FIGURE 11: Comparison of the MSEs and the CRB in estimating DOA3 (three sources case).

\section{CONCLUSION}

In this paper, we address the problem of maximum likelihood DOA estimation of multiple wideband sources in the presence of nonuniform sensor noise. A new closed-form expression of the CRB has been derived in this paper, and the performance of DOA estimation using UCA has been studied.

Unlike the ML estimator under the uniform white noise assumption, no separable solution for DOA estimation seems to exist in the nonuniform noise case. We present two processing algorithms which approach this problem from different directions. The SC-ML implements an iterative procedure which stepwise concentrates all the nuisance parameters numerically. The AC-ML is noniterative and seeks to optimize over an approximately concentrated log-likelihood function. Computer simulations have shown that the SC-ML requires only a few iterations to converge in all scenarios, and the AC-ML consistently gives better performance than the 1st-iteration estimate of the SC-ML. Both the SC-ML and AC-ML show significant improvement over the conventional uniform-ML estimator and attain a solution close to the derived CRB in the high SNR region.

\section{APPENDICES}

\section{A. DERIVATION OF CRB}

From the deterministic wideband signal model (2), the $(i, j)$ th element of the Fisher information matrix (FIM) can be expressed as

$$
\begin{aligned}
{[\mathbf{F}]_{i, j}=} & \frac{N}{2} \operatorname{tr}\left\{\mathbf{Q}^{-1} \frac{\partial \mathbf{Q}}{\partial \psi_{i}} \mathbf{Q}^{-1} \frac{\partial \mathbf{Q}}{\partial \psi_{j}}\right\} \\
& +2 \Re\left\{\frac{\partial \mathbf{b}^{H}}{\partial \psi_{i}}\left(\mathbf{I}_{N / 2} \otimes \mathbf{Q}^{-1}\right) \frac{\partial \mathbf{b}}{\partial \psi_{j}}\right\},
\end{aligned}
$$

where $i, j=1, \ldots,(M+M N+P) \cdot \psi_{i}$ is the $i$ th element of $\Psi$, and $\mathbf{b}$ is an $N P / 2 \times 1$ vector defined as

$$
\mathbf{b}=\left[\mathbf{S}(1)^{T} \mathbf{D}(1)^{T}, \ldots, \mathbf{S}\left(\frac{N}{2}\right)^{T} \mathbf{D}\left(\frac{N}{2}\right)^{T}\right]^{T}
$$

Define

$$
\mathbf{H}(k)=\left[\begin{array}{cccc}
S^{(1)}(k) & & & 0 \\
& S^{(2)}(k) & & \\
& & \ddots & \\
0 & & & S^{(M)}(k)
\end{array}\right],
$$

then after some manipulations we have

$$
\begin{aligned}
\frac{\partial \mathbf{b}^{H}}{\partial \boldsymbol{\Theta}} & =\left[\mathbf{H}^{H}(1) \mathbf{E}^{H}(1), \ldots, \mathbf{H}^{H}\left(\frac{N}{2}\right) \mathbf{E}^{H}\left(\frac{N}{2}\right)\right], \\
\frac{\partial \mathbf{b}^{H}}{\partial \mathbf{S}_{R}(k)} & =\left[\mathbf{e}_{k} \otimes \mathbf{D}(k)\right]^{H}, \\
\frac{\partial \mathbf{b}^{H}}{\partial \mathbf{S}_{I}(k)} & =-j\left[\mathbf{e}_{k} \otimes \mathbf{D}(k)\right]^{H},
\end{aligned}
$$

where $\mathbf{S}_{R}(k)$ and $\mathbf{S}_{I}(k)$ denote the real and imaginary part of $\mathbf{S}(k)$, respectively, and $\mathbf{e}_{k}$ is the vector containing a one in the 
$k$ th position and zeros elsewhere. From (A.1) and (A.4), we can derive the submatrices of the FIM as follows:

$$
\begin{aligned}
& \mathbf{F}_{\Theta \Theta}=2 \Re\left\{\sum_{k=1}^{N / 2} \mathbf{H}^{H}(k) \widetilde{\mathbf{E}}^{H}(k) \widetilde{\mathbf{E}}(k) \mathbf{H}(k)\right\}, \\
& \mathbf{F}_{\Theta \mathbf{S}_{R}(k)}=2 \mathfrak{R}\left\{\sum_{k=1}^{N / 2} \mathbf{H}^{H}(k) \widetilde{\mathbf{E}}^{H}(k) \widetilde{\mathbf{D}}(k)\right\}, \\
& \mathbf{F}_{\Theta \mathbf{S}_{I}(k)}=2 \mathfrak{I}\left\{\sum_{k=1}^{N / 2} \mathbf{H}^{H}(k) \tilde{\mathbf{E}}^{H}(k) \tilde{\mathbf{D}}(k)\right\}, \\
& \mathbf{F}_{\mathbf{S}_{R}(k) \Theta}=2 \Re\left\{\sum_{k=1}^{N / 2} \widetilde{\mathbf{D}}^{H}(k) \widetilde{\mathbf{E}}(k) \mathbf{H}(k)\right\}, \\
& \mathbf{F}_{\mathbf{S}_{I}(k) \Theta}=-2 \mathfrak{I}\left\{\sum_{k=1}^{N / 2} \widetilde{\mathbf{D}}^{H}(k) \widetilde{\mathbf{E}}(k) \mathbf{H}(k)\right\}, \\
& \mathbf{F}_{\mathbf{S}_{R}\left(k_{1}\right) \boldsymbol{S}_{R}\left(k_{2}\right)}=2 \mathfrak{R}\left\{\tilde{\mathbf{D}}^{H}\left(k_{1}\right) \tilde{\mathbf{D}}\left(k_{2}\right)\right\} \delta_{k_{1}, k_{2}}, \\
& \mathbf{F}_{\mathbf{S}_{I}\left(k_{1}\right) \mathbf{s}_{I}\left(k_{2}\right)}=2 \mathfrak{R}\left\{\tilde{\mathbf{D}}^{H}\left(k_{1}\right) \tilde{\mathbf{D}}\left(k_{2}\right)\right\} \delta_{k_{1}, k_{2}} \text {, } \\
& \mathbf{F}_{\mathbf{S}_{R}\left(k_{1}\right) \mathbf{s}_{I}\left(k_{2}\right)}=2 \mathfrak{I}\left\{\tilde{\mathbf{D}}^{H}\left(k_{1}\right) \tilde{\mathbf{D}}\left(k_{2}\right)\right\} \delta_{k_{1}, k_{2}} \text {, } \\
& \mathbf{F}_{\mathbf{s}_{I}\left(k_{1}\right) \boldsymbol{s}_{R}\left(k_{2}\right)}=-2 \mathfrak{I}\left\{\tilde{\mathbf{D}}^{H}\left(k_{1}\right) \tilde{\mathbf{D}}\left(k_{2}\right)\right\} \delta_{k_{1}, k_{2}}, \\
& \mathbf{F}_{\mathbf{q q}}=\frac{N}{2} \mathbf{Q}^{-2},
\end{aligned}
$$

where $\delta_{k_{1}, k_{2}}=1$ if $k_{1}=k_{2}$, and 0 otherwise. The cross terms in the information matrix between the signal and noise parameters are all zero.

Let us define the following matrices:

$$
\begin{aligned}
& \mathbf{F}_{\Theta \mathbf{S}}=\mathbf{F}_{\mathbf{S} \Theta}^{T}=\left[\mathbf{F}_{\Theta \mathbf{S}(1)}, \mathbf{F}_{\Theta \mathbf{S}(2)}, \ldots, \mathbf{F}_{\Theta \mathbf{S}(N / 2)}\right], \\
& \mathbf{F}_{\mathbf{S S}}=\left[\begin{array}{ccc}
\mathbf{F}_{\mathbf{S}(1) \mathbf{S}(1)} & \mathbf{0} \\
& \ddots & \\
\mathbf{0} & & \mathbf{F}_{\mathbf{S}(N / 2) \mathbf{S}(N / 2)}
\end{array}\right],
\end{aligned}
$$

where

$$
\begin{aligned}
& \mathbf{F}_{\boldsymbol{\Theta S}(k)}=\mathbf{F}_{\mathbf{S}(k) \Theta}^{T}=\left[\mathbf{F}_{\boldsymbol{\Theta} \mathbf{S}_{R}(k)}, \mathbf{F}_{\Theta \mathbf{S}_{I}(k)}\right], \\
& \mathbf{F}_{\mathbf{S}(k) \mathbf{S}(k)}=\left[\begin{array}{ll}
\mathbf{F}_{\mathbf{S}_{R}(k) \mathbf{S}_{R}(k)} & \mathbf{F}_{\mathbf{S}_{R}(k) \mathbf{S}_{I}(k)} \\
\mathbf{F}_{\mathbf{S}_{I}(k) \mathbf{S}_{R}(k)} & \mathbf{F}_{\mathbf{S}_{I}(k) \mathbf{S}_{I}(k)}
\end{array}\right] .
\end{aligned}
$$

Then the FIM matrix can be expressed as

$$
\mathbf{F}=\left[\begin{array}{ccc}
F_{\Theta \Theta} & F_{\Theta S} & 0 \\
F_{S \Theta} & F_{S S} & 0 \\
0 & 0 & F_{\mathrm{qq}}
\end{array}\right]
$$

Applying the partitioned inversion formula, the inverse of the CRB matrix for $\Theta$ can be obtained by

$$
\begin{aligned}
\mathbf{C R B}_{\Theta \Theta}^{-1} & =\mathbf{F}_{\Theta \Theta}-\mathbf{F}_{\Theta \mathbf{S}} \mathbf{F}_{\mathbf{S S}}^{-1} \mathbf{F}_{\mathbf{S} \Theta} \\
& =\mathbf{F}_{\Theta \Theta}-\sum_{k=1}^{N / 2} \mathbf{F}_{\Theta \mathbf{S}(k)} \mathbf{F}_{\mathbf{S}(k) \mathbf{S}(k)}^{-1} \mathbf{F}_{\mathbf{S}(k) \Theta}
\end{aligned}
$$

Using (A.5), and the following complex multiplication rules:

$$
\begin{aligned}
\mathfrak{I}\{\mathbf{Y}\} \mathfrak{R}\left\{\mathbf{Z}^{H}\right\}+\mathfrak{R}\{\mathbf{Y}\} \mathfrak{I}\left\{\mathbf{Z}^{H}\right\}=\mathfrak{I}\left\{\mathbf{Y} \mathbf{Z}^{H}\right\}, \\
\mathfrak{R}\{\mathbf{Y}\} \mathfrak{R}\left\{\mathbf{Z}^{H}\right\}-\mathfrak{I}\{\mathbf{Y}\} \mathfrak{I}\left\{\mathbf{Z}^{H}\right\}=\mathfrak{R}\left\{\mathbf{Y} \mathbf{Z}^{H}\right\},
\end{aligned}
$$

one can easily show that

$$
\begin{aligned}
\mathbf{C R B}_{\Theta \Theta}^{-1} & =2 \Re\left\{\sum_{k=1}^{N / 2} \mathbf{H}^{H}(k) \widetilde{\mathbf{E}}^{H}(k) \mathbf{P}_{\widetilde{\mathbf{D}}(k)}^{\perp} \widetilde{\mathbf{E}}(k) \mathbf{H}(k)\right\}, \\
& =2 \Re\left\{\sum_{k=1}^{N / 2}\left[\widetilde{\mathbf{E}}^{H}(k) \mathbf{P}_{\widetilde{\mathbf{D}}(k)}^{\perp} \widetilde{\mathbf{E}}(k)\right] \odot \mathbf{R}_{\mathbf{S}}^{T}(k)\right\} .
\end{aligned}
$$

\section{B. PROOF OF THE $180^{\circ}$ PERIODICITY OF THE SINGLE SOURCE NONUNIFORM CRB}

Consider the signal model of Section 2 in the single source case, the $p$ th element of $\widetilde{\mathbf{d}}(k)$ and $\tilde{\mathbf{e}}(k)$ can be written as

$$
\begin{aligned}
& {[\tilde{\mathbf{d}}(k)]_{p}=q_{p}^{-1 / 2} e^{-j 2 \pi k t_{p} / N},} \\
& {[\tilde{\mathbf{e}}(k)]_{p}=q_{p}^{-1 / 2}\left(\frac{-j 2 \pi k}{N}\right) \dot{t}_{p} e^{-j 2 \pi k t_{p} / N},}
\end{aligned}
$$

where

$$
\begin{aligned}
& t_{p}=-\frac{F_{s} r_{p}}{v} \cos \left(\theta-\phi_{p}\right), \\
& \dot{t}_{p}=\frac{d t_{p}}{d \theta}=\frac{F_{s} r_{p}}{v} \sin \left(\theta-\phi_{p}\right) .
\end{aligned}
$$

It follows that

$$
\begin{aligned}
\|\tilde{\mathbf{d}}(k)\|^{2} & =\sum_{p=1}^{P} q_{p}^{-1}, \\
\|\tilde{\mathbf{e}}(k)\|^{2} & =\sum_{p=1}^{P} q_{p}^{-1}\left(\frac{2 \pi k r_{p} F_{s}}{N v}\right) \sin ^{2}\left(\theta-\phi_{p}\right), \\
\left|\tilde{\mathbf{d}}(k)^{H} \tilde{\mathbf{e}}(k)\right|^{2} & =\left|\sum_{p=1}^{P} q_{p}^{-1}\left(\frac{2 \pi k r_{p} F_{s}}{N v}\right) \sin \left(\theta-\phi_{p}\right)\right|^{2} .
\end{aligned}
$$

Substituting (B.3) into (39), it is clear that

$$
\mathrm{CRB}^{-1}(\theta)=\mathrm{CRB}^{-1}(\theta+\pi) .
$$

\section{ACKNOWLEDGMENTS}

This work was partially supported by NSF CENS program, NSF Grant EF-0410438, AROD-MURI PSU Contract 50126, and ST Microelectronics, Inc.

\section{REFERENCES}

[1] R. Schmidt, A signal subspace approach to multiple emitter location and spectral estimation, Ph.D. thesis, Stanford University, 1981.

[2] M. Wax and T. Kailath, "Optimum localization of multiple sources by passive arrays," IEEE Transactions on Acoustics, Speech, and Signal Processing, vol. ASSP-31, no. 5, pp. 12101218, 1983. 
[3] T. Kailath and R. Roy, "Estimation of signal parameters via rotational invariance techniques," IEEE Transactions on Acoustics, Speech and Signal Processing, vol. 37, pp. 984-995, 1989.

[4] P. Stoica and A. Nehorai, "Music, maximum likelihood and cramer-rao bound," IEEE Transactions on Acoustics, Speech and Signal Processing, vol. 37, pp. 720-741, 1989.

[5] J. F. Boehme, "Estimation of spectral parameters of correlated signals in wavefields," Signal Processing, vol. 11, no. 4, pp. 329337, 1986.

[6] P. Stoica and A. Nehorai, "Performance study of conditional and unconditional direction-of-arrival estimation," IEEE Transactions on Acoustics, Speech, and Signal Processing, vol. 38, no. 10, pp. 1783-1795, 1990.

[7] J. F. Boehme, "Separated estimation of wave parameters and spectral parameters by maximum likelihood," in Proceedings of the IEEE International Conference on Acoustics, Speech and Signal Processing (ICASSP '86), pp. 2819-2822, Tokyo, Japan, 1986.

[8] A. G. Jaffer, "Maximum likelihood direction finding of stochastic sources: a separable solution," in Proceedings of the IEEE International Conference on Acoustics, Speech and Signal Processing (ICASSP '88), pp. 2893-2896, New York, NY, USA, 1988.

[9] P. Stoica and A. Nehorai, "On the concentrated stochastic likelihood function in array signal processing," Circuits, Systems, and Signal Processing, vol. 14, no. 5, pp. 669-674, 1995.

[10] H. Wang and M. Kaveh, "Coherent signal-subspace processing for the detection and estimation of angles of arrival of multiple wide-band sources," IEEE Transactions on Acoustics, Speech, and Signal Processing, vol. 33, no. 4, pp. 823-831, 1985.

[11] P. M. Schultheiss and M. Messer, "Optimal and suboptimal broad-band source location estimation," IEEE Transactions on Signal Processing, vol. 41, no. 9, pp. 2752-2763, 1993.

[12] J. C. Chen, R. E. Hudson, and K. Yao, "Maximum-likelihood source localization and unknown sensor location estimation for wideband signals in the near-field," IEEE Transactions on Signal Processing, vol. 50, no. 8, pp. 1843-1854, 2002.

[13] J. P. Le Cadre, "Parametric methods for spatial signal processing in the presence of unknown colored noise fields," IEEE Transactions on Acoustics, Speech, and Signal Processing, vol. 37, no. 7, pp. 965-983, 1989.

[14] B. Friedlander and A. J. Weiss, "Direction finding using noise covariance modeling," IEEE Transactions on Signal Processing, vol. 43, no. 7, pp. 1557-1567, 1995.

[15] V. Nagesha and S. Kay, "Maximum likelihood estimation for array processing in colored noise," IEEE Transactions on Signal Processing, vol. 44, no. 2, pp. 169-180, 1996.

[16] A. Paulraj and T. Kailath, "Eigenstructure methods for direction of arrival estimation in the presence of unknown noise fields," IEEE Transactions on Acoustics, Speech, and Signal Processing, vol. 34, no. 1, pp. 13-20, 1986.

[17] J. M. Velni and K. Khorasani, "Localization of wideband sources in colored noise via Generalized Least Squares (GLS)," in Proceedings of the IEEE Workshop on Statistical Signal Processing, pp. 525-529, 2005.

[18] M. Pesavento and A. B. Gershman, "Maximum-likelihood direction-of-arrival estimation in the presence of unknown nonuniform noise," IEEE Transactions on Signal Processing, vol. 49, no. 7, pp. 1310-1324, 2001.

[19] M. Wax and T. Kailath, "Detection of signals by information theoretic criteria," IEEE Transactions on Acoustics, Speech and Signal Processing, vol. 33, pp. 387-392, 1985.

[20] I. Ziskind and M. Wax, "Maximum likelihood localization of multiple sources by alternating projection," IEEE Transactions on Acoustics, Speech, and Signal Processing, vol. 36, no. 10, pp. 1553-1560, 1988.

[21] K. Sharman, "Maximum likelihood estimation by simulated annealing," in Proceedings of the IEEE International Conference on Acoustics, Speech and Signal Processing (ICASSP '88), pp. 2741-2744, New York, NY, USA, April 1988.

[22] P. Stoica and A. B. Gershman, "Maximum-likelihood DOA estimation by data-supported grid search," IEEE Signal Processing Letters, vol. 6, no. 10, pp. 273-275, 1999.

[23] S. Kay and S. Saha, "Mean likelihood frequency estimation," IEEE Transactions on Signal Processing, vol. 48, no. 7, pp. 19371946, 2000.

[24] M. Li and Y. Lu, "Genetic algorithm based maximum likelihood DOA estimation," in IEE Conference Publication, vol. 490, pp. 502-506, October 2002.

[25] P. J. Chung and J. F. Böhme, "DOA estimation using fast EM and SAGE algorithms," Signal Processing, vol. 82, no. 11, pp. 1753-1762, 2002.

[26] L. Yip, J. C. Chen, R. E. Hudson, and K. Yao, "Numerical implementation of the AML algorithm for wideband DOA estimation," in Proceedings of SPIE, vol. 5205, pp. 164-172, August 2003.

[27] Y. Bresler and A. Macovski, "Exact maximum likelihood parameter estimation of superimposed exponential signals in noise," IEEE Transactions on Acoustics, Speech, and Signal Processing, vol. 34, no. 5, pp. 1081-1089, 1986.

[28] P. Stoica and K. C. Sharman, "Novel eigenanalysis method for direction estimation," in IEE Proceedings. Part F. Communications, Radar and Signal Processing, vol. 7, pp. 19-26, February 1990.

[29] L. Yip, J. C. Chen, R. E. Hudson, and K. Yao, "Cramér-Rao bound analysis of wideband source localization and DOA estimation," in Proceedings of SPIE, vol. 4791, pp. 304-316, August 2002.

[30] A. L. Matveyev, A. B. Gershman, and J. F. Böhme, "On the direction estimation Cramer-Rao bounds in the presence of uncorrelated unknown noise," Circuits, Systems, and Signal Processing, vol. 18, no. 5, pp. 479-487, 1999.

[31] M. Wax, J. Sheinvald, and A. J. Weiss, "Detection and localization in colored noise via generalized least squares," IEEE Transactions on Signal Processing, vol. 44, no. 7, pp. 1734-1743, 1996.

[32] P. Stoica and R. Moses, Spectral Analysis of Signals, PrenticeHall, Englewood Cliffs, NJ, USA, 2005.

[33] U. Baysal and R. L. Moses, "Optimal array geometries wideband DOA estimation," in Proceedings of the MSS Meeting on Battlefield Acoustic and Seismic Sensors, Laurel, Md, USA, October 2001.

[34] S. M. Kay, Fundamentals of Statistical Signal Processing: Estimation theory, Prentice-Hall, Englewood Cliffs, NJ, USA, 1993. 